\title{
Ingeborg Maus Sinn und Bedeutung von Volkssouveränität in der modernen Gesellschaft
}

Auf den ersten Blick scheint der Begriff der Volkssouveränität zu jenen klassischen Begriffen der Demokratietheorie zu gehören, die in der gegenwärtigen Gesellschafı keine mögliche Realitäı mehr bezeichnen. Dies gilt nicht nur für den Begriff im ganzen, sondern auch für seine Bcstandteile. "Souveränität" scheint durch die Tarsache widerlegr zu scin, daß politische Entscheidungsprozesse zunehmend mit ihren gesellschaftlichen Regelungsbereichen in einer Weise vernetzr sind, daß eindeurige Zuständigkeiten und Verantwortlichkeiten überhaupt nicht mehr ausfindig zu machen sind. Erst recht kann dem Volksbegriff angesichts der heutigen Regionalisierung und Pluralisierung aller gesellschaftlichen Problemlagen und der fortgeschrittenen Entwicklung zu einer multikulturelien Gesellschaft kcine kompaktc Bedeutung mehr zugeordnet werden. Schließlich entbehrten Konzeptionen des „Volkswillens - ohne die eine Theorie der Volkssouveränität nicht auskommen kann -, soweit sie ein kollektives Entscheidungssubjekt unterstellen, schon immer der gesellschafilichen Grundlage. Sie waren aber, was noch zu untersuchen ist, auch in den klassischen Demokratictheorien der Aufklärung so nicht gemeint.

Es scheint jedenfalls der akuellen gesellschaftlichen Situacion zu entsprechen, daß der Begriff der Volkssouvcránität cincm kollektiven Verdrängungsprozeß unterliege - ein Phänomen, das innerhalb der bundesrepublikanischen Diskussion in konservativen und linken bzw. walternativen "Stellungnahmen gleichermaßen beherrschend ist. Typisch für die in der Bundesrcpublik entwickelte Rechts- und Vcrfassungstheorie ist die Auffassung, daß die Geltung einer positivrechtlichen Verfassung die Existenz jedes Souveräns, auch eines demokratischen Souveräns, ausschließe und Volkssouveränität nur als Prämisse der gettenden Vcrfassung zu verstehen sei.' Volkssouveränität wird so auf einen einmaligen Akt der verfassunggebenden Gewalt des Volkes reduzierr, der sich in dieser eher symbolischen Bedeutung erschöpft und einc Verfassung konsticuiert, der zunchmend selbst "Souveränität \& zugeschrieben wird. ${ }^{2}$ Diese herrschende Verfassungstheorie kennt weder den gesellschaftlichen "Ortx noch das Subjekt der Souveränität und entspricht in dieser Hinsichı den faktischen Vernetzungen und der systemischen Zirkularirät der heutigen politischen Entscheidungsprozesse. Sie folgt der gleichen Logik, mit der Niklas Lutimann formuliert, daß nicht mehr einzclne Entscheidungssubjekte und gar nicht mehr "das

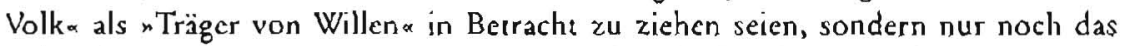
politische System in seiner Gesamtheit.' Während diese konservativen Fassungen des Problems daraul hinauslaufen, eine unterdeterminierte Souveränität den institu-

1 Marun Knele, Finführung in dic Staatslehrc. Die geschichtlichen Legiumutäsgrundlagen des demokratsscben Verfassungsstaates, Reinbek 1975, S. 1 is if.

2 Ebd. S. $1061 f ., 224$ ff.

3 Niklas Luhmann, Legummason durch Verfahren, Neuwied/Berlin rgbg, S. is 3 f. und Anm. s. 
tionalisierten und systemisch strukrurierten Organisationskomplexen insgesamt zuzuordnen, denen gegenüber der cinstige demokratische Souverän zum »Publikum " degeneriert", das aus eincm autonomen, $d$. $h$. wesentlich von konkretem Konsens unabhängigen System heraus mit unzurechenbaren Entschcidungen versorgt wird, wiederholen alternative Konzeptionen die gleiche Dichotomie aus umgekehreer Perspektive.

Der Procest gegen die selbstreferentiellc Versclbständigung politischer Entscheidungsprozesse aurde von den neucn basisdemokratischen Bewegungen bezcichnenderweise nicht als Ausübung von Volkssouveränität verstanden, sondern klcidete sich in das Gewand des Widerstandsreches oder des bürgerlichen Ungehorsams. Damit ist keineswegs ein nur terminologischer Unterschied bezeichnet. Wälurend die Demokratictheoric der Aufklärung eine umfassende Handlungskompetenz des "souveränen Volkes« begründete, die das Volk gleichzeirig als Hürer der bestehenden Verfassung und als permanene wirksame verfassunggebende Gewalt einsetztes, also die Entscheidung über Fortbestand und Innovation an der gesellschaftlichen Basis verortete, setzen Widerstand bzw. Ungehorsam ein lediglich reaktives Verhalten von Rechtsadressaten auf vorgefertigte rechthiche und politische Entscheidungen voraus. Gegen Innovationen von oben mobilisieren sich Widerstand und Verweigerung von unten. Dicses alternarive Konzept enthält darum nicht wesentlich mehr als die Systemtheorie der gcsellschaftlichen Basis ohnehin noch zubilligt. Es näherr sich jener Residualkatcgoric »basaler Souveränitzär ${ }^{6}{ }^{6}$ an, die Luhmann zufolge lediglich die Möglichkeir bezeiclıner, sich in alläglichen Interaktionen auf vorgegebene Rechtsentscheidungen zu beziehen oder nicht zu bezichen. Ebenso nehmen sich "Widerstand" und "Ungehorsam "lediglich die - wenn auch unallräglich und demonstrativ vertretene - Freiheit, geserztes Recht nicht anzuerkennen. Dic von Luhmann bezeichnete Dichotomie zwischen einer systemischen Entscheidungssouveränität und ciner lediglich negatorischen basalen Souveräniät liegt darum auch den gegenwärtig vorherrschenden alternativen Konzepren zugrunde.

In ähnlicher Weise konvergieren die alternativen Selbstbeschreibungen des Ungehorsams mir der herrschenden Rechts- und Verfassungstheoric, dercn Exorzismus jeglicher Volkssouveränität im Zcichen der Reches- und Verfassungssouveränitär ganz offenkundig ist. Auch wo basisdemokratische Initiativen tatsächlich nach Prinzipien der Volkssouveränität verlahren, indem sie politische Innovationen und Rechtsänderungen aus der Perspektive "von unten « zu bewirken suchen, subsumieren sie ihr Selbstverständnis und oft auch ihre Praxis der herrschenden justizstaatlichen Doktrin. Selbst genuin liberale rechrswissenschaftliche Argumentationen, die bereit sind, diese Basisbewegungen zu unterstürzen, verstärken diesen Effekt. So läufr die Praxis vieler Protestbewegungen darauf hinaus, durch synbolische Regelverstöße cine gerichtliche Klärung der Rechtslage zu bervirken; und dic unterstürzende juristische Theorie definierc schließlich "die Bereitschalt, sich einem Gcrichrsverfahren zu stellen, ... (ais ein) wesentliches Element des zivilen Ungehorsams «.? Auf diese Weise sind Widerstand und bürgerlicher Ungehorsam nichr

4 Niklas Luhmann, Cirundrechic zls Instruution, Berlin 1969. S. 1 s5: ders., Legiumation durch Verfahren (Anm. 3), S. 19x.

J John Locke, Two Treauses of Government. Hg. Peter Lasletr. Cambridge 2. Aufl. 1967, 168, - Jean-

J. Rousseau, Du contrat social on principes du drou polisique. Edicion Gamser, Pans 1963, II, 18 ; Emmanuel Joseph Sieyes, Was use der Dntte Stand?, in: Ders., Poliusche Schriften 1788-1790, $\mathrm{Hg}_{\mathrm{g}}$ Eberhard Schmile, Damstadu/Neuwied 1975, S. 117ff., 1646 if.

6 Niklas Luhmann, Ausdifferenzerung des Rechts, FrankfundM. 1981, S. $3^{8}$

7 Ralf Dreier, Widerstand und avilce Ungehorsam im Rechessiale, in: Peter Gloız (Hg), Zivilcr

Ungehorsam im Rechessesat, Frankfund M. $1983.8_{3}$. ${ }_{4} f_{1}, 62$. 
auslösende Momente cines demokratischen Willensbildungsprozesses, der zu einer generellen Geserzesänderung fülurte, sondern erschöpfen sich darin, den "Rechtsweg e einzuleiten. ${ }^{\vee}$ Dic gcrichtsförmige Bearbeitung einer solchen Konflikclage führ aber, sofern nicht glcichzeitig einc öflentliche Diskussion in Gang gesctzt wird, die ihrcrseits demokratisch motivicrte Entscheidungen bewirkt, weder zu einer Rechtsanderung noch zu einer ${ }^{\circ}$ Klärung ${ }^{2}$ des Rechts. Angesichts des gegenwärtigen Merhodenkanons der Rechtsprechung, der äußerst situative Abwägungen zuläßt und es den Gerichten erlaubt, vergleichbare Falle als je neuc zu definieren", betrifft eine gerichtsförmige Entscheidung immer nur den vorliegenden Einzelfall. Gleichzeitig kommt es zu einer Juridifizierung der basisdemokratischen Aktivitäten, zu dem erklärten Versuch, bürgerlichen Ungehorsam wiederum »an Regeln zu binden $\alpha^{10}-$ Regeln, die in jeder einzelnen Sizuation von den Gerichten neu bestimmt verden können.

Nur die extreme Situativität in der gegenwärtigen Vcrrechtlichung von zivilem Ungchorsam und Widerstand trennt aber diese noch von einer schlichten Wiederbeicbung des Widerstandsrechts als eines feudaiständischen Rechtsinstiturs. W/ss Volkssouveränizät von Widerscandsrcchi, das sie cinmal hiscorisch ablöste, unterscheidet, ist gerade die Tatsache, daß sie sich nicht aus bestehendem Recht oder einer geltenden Verfassung ableiter, sondem der gesanten Rechtsordnung vorausliegr und diese erst begründet. Souveränität bedeutete auch in der Konnotacion der "Volkssouveränität« einc legibus-solutus-Posizion, dic die Aufklärungsphilosophic in der Forderung permanenter Verfassungsrevision durch das „Volk « arcikulierte und in der Formel erläucerte, daß in dieser Hinsichr nur die Regierung, nicht aber das Volk an die Verfassung gebunden sei." Das mitrelalıerliche Widerstandsrecht dagegen war tatsächlich ein Rechesinstitut, bezcichnete also eine institurionalisierte, kodifizierte und justiziable Funktion und bezog sich auf eine prinzipicll unverfügbarc Rechtsordnung. Hier war jede gesellschaftlich-politische Aktion nur aus der vorgegebenen Rechtsordnung ableitbar, die sowohl zu ihrer Interpretation wie zu ihrer Exckution Gerechrigkeitsexpertokratien begründete. Unter Berufung auf diescs Widerstandsrecht wurde von etablierten Instanzen altes Privilegienrecht gegen aufkommende absolutistische Innovationen verteidigt." Gerade die Tatsache, daß seit dem ausgehenden Hochmictelalter Gesetzgebungsansprüche der Herrscher sich ankündigten ${ }^{13}$, machte Widerscand im Namen eines Rechts plausibel, das Priorität vor jeder Handlung und Entscheidung beanspruchte. Auch der ständestaatliche Kompromiß, in dem später die absolucistischen Tendenzen zur souveränen Rechtsetzung mit Institutionen zur Wahrung des alten Rechts und der privilegieren Freiheiten zusammengeschaltet wurden, ließ nur dic Idee der Prioritär des Rechts vor der Souveränität der Rechtsetzung zu. Weil nämlich in diescm widersprüchlichen Kompromiß Ort und Subjekt der Souveränität nicht zu bescimmen waren, erhielt sich die tradierte mittelalterliche Vorstellung einer Souveränität der Gesamtheit der politischen Institutionen und einer Souveränität der Rechtsordnung als

8 A. a. O., S. 73 .

9 S. mu Nachwesen: Ingeborg Maus, Zur Problematik des Rationalitúts-und Rechesstaatsposulats in der gegenwänigen jurıseıschen Methodik am Bespiel Friedrich Müllers, in: Wolfgang Abendroth u. 2. Ordnungsmache? Uber das Verhältnis von Legalitae, Konsens und Herrschalt - Festschrift für Helmut Ridder zum 60. Gcburtseag. FrankfureM. 1981, S. 1536í.

10 Ralf Dreser, Widerseand (Anm. 7), S. 73.

11 Emmanuel J. Sicyès, Was ist der Drite Stand (Anin. S), S. 167

Is Vgl. z. B. Fritz Kern, Goutesgnadentum und Widerstandsrecht im fruhen Mituelalicr, 7. Aull. Darmstad 1980, S. 227 fl; Kurr Wolxendorff, Staatsrecht und Vaturreche in der Lehre vom Widerstandsrecht des Volkes gegen rechiswidnge Ausubung der Stantsgewall, Neudruck Aalen 1961.

13 Sien Gagnér, Studien zur Ideengeschichte der Gesetzgcbung, Stockholm, Uppsala, Gáteborg 1960, S. 118,179 fi., 34111 . 
solcher aufrecht." So argumentierte bekanndich noch Coke in den englischen Konfliksen des 17.Jahrhunderts anläßlich seiner generalisierenden Interpretacion der feudalen Freiheitsverbrielung der Magna Charta, daß diese keinen Souverän dulde, weil die Magna Charra selbst zusammen mit den gettenden Statutes "absolut * sei."

Es ist vor allem diese Vorstellung einer Souveränität des Rechts bzw. der Verfassung und nicht ctwa des *Volkes $\propto$, die die gegenwärtige Renaissance des Widerstandsrechrs tarsächlich zu einer Wiederkehr des Vergangenen machr. Die Praxis und noch meir die Selbstinterpretation der basisdemokratischen Bewegungen stützen selber den herrschenden Trend, gegen den sie sich wenden, indem sie sich in die bescehende Dichoromie zwischen systemisch verselbständigten Entscheidungsprozessen und bloß negatorischer gesellschaftlicher Reaktion einfügen und sich zur Artikulation ihrer Verweigerungen Aktionsformen bedicnen, die im Ergebnis die herrsthende justizstaatliche Entwicklung bestärigen. Auf diese Weise wird die Idee, daß nur demokratisch gesecztes Recht legitim sei, verabschieder und die Initiative der Rechtsentwicklung an die Gerichtc zurückgegcben. Diese trneute Annäherung an mittelalıerliche Rechtsverhälınisse bricht zugleich mit allen Prinzipien demokratischer Legitimation des politischen Handelns. Dieses versteht sich nicht mehr als Vollzug der Ergebnisse basisdemokmtischer und institutionelI-demokratischer Mejnungs- und Willensbildungsprozcssc, die sich lediglich im Rahmen der verfassungsnäßigen Verfahrensbestimmungen bzw. in den von Frcihcitsrecheen ausgegrenzren autonomen Handlungsbereichen entwickeln, sondern als unmittelbare Exekution von Verfassungsinhaleen, die gegenüber demokrarischem Prozcdere "vorkonsentiert" sind. ${ }^{16}$ Demokratische Willensbildung wird folgerichtig durch die Interpreiation "souveräner ", vorgegebener Verfassungsinhalte ersecz $t$ ", in der das Verfassungsgericht und die Instanzgerichre qua experrokratischer Kompecenz gegenüber alternativen gesellschaftlichen Interpretationsbemühungen stets das letzre Wort behalten. In dicser Rückkehr zur Herrschaft der Exegese wird die Existenz weiter Spielräume der Ver/assungsinterpretation damit gerechtfertigt, daß die Verfassung der Dynamik gesellschafticher Entwicklung jewcils angepaßt werden müsse. Hatte die europäische Demokratiecheoric des 58 . Jahrliunderts noch darauf bestanden, daß dies Aufgabe der permanenten verfassunggebenden Gewalt des Volkes sei, so ist dieser wesentliche Aspekt von Volkssouveränität heute durch die Verfassungsgerichtsbarkeit usurpiert.

Eine Deutung dieser Entwicklungstrends, die cine Refeudalisierung der politischen Integrationsmuster diagnostizierr, wird allerdings nicht umbin können, sich mit dem Anpassungsdruck zu beschärigen, der von fakrischen gesellschaftlichen Vcränderungen ausgehend auf die moderne Konzepzion demokratischer Insritutionalisierung einwirke und deren Basisprinzip der Volkssouvcränität zum Verschwinden bringt. Es geht deshaib im folgenden darum, die Qualität dieser gesellschaflichen Veränderungen und den Charakter des gegenwärtigen Umbaus der politischen

14 Vgl. Christopher Hill, The Century of Revolution 1603-1714, New York 1966, S.65f.

is Zitien nach J.W. Gough, Fundamental Law in English Consututional Hisiory, 2. Aufl. Oxford 196r, S.64.

16 Der Begriff xvorkonsenuerı \& bezculnet ein demokrauecheoreusches Pendant an jener Moralkonzepuon, dic Habermas als morologische kritusien, weil ste eine transzendentale oder sogar substannelle Vorverstündigung untersiellt, die den intersubjektıven öffentlichen Diskurs überflussıg machti s. $x$. B. Jurgen Habermas, Moraltäı und Sieclichkete. Treffen Hegels Einwände gegen Kant auch aul dic Diskurserhik zu in: Wolfgang Kuhlmann ( $\mathrm{Hg}$.), Moralirät?, und Sitclichken, FrankfuruM. 1986. S. 16 ff., 24 1.

17 Peter Haberle, Dic offene Gesellschafe der Verfassungsinterpreten, in: Drs.. Verfassung als óffendicher ProztB. Materiatien zu ener Verfassungstheone der offenen Gesellschaft. Berlin 1978. S. Iss ff. 
Entscheidungsprozesse näher einzuschätzen (I). Soweit dabci die These vertreten wird, daß gegenwärtig "eindimensionale institurionellc und cheorecische Anpassungslcistungen überwiegen, ist im weireren der Versuch uncernommen, eine andere Arr dor Anpassung zu erörtern, die veränderte gesellschaftliche Kontexte berücksichtigt, ohne die wesentlichen Emanzipationspotentiale klassischer Demokratietheoric zugunsten vordemokratischer Integrationsmuster aufzugeben. Dieser Versuch konzentriert sich auf eine Rekonstruktion von Volkssouveränität unter gewandelten Bedingungen (II).

\section{I.}

Eine überaus kompetente Analyse des gegenwärrigen gesellschaftlichen Umbruchs von Ulrich Beck betrifft die Strukturen der "Risikogesellschaft ". ${ }^{8} \mathrm{Da}$ zugleich einige ihrer politisch-institurionellen Folgerungen für dic heute herrschenden Anpassungstendenzen reprásencariv zu sein scheinen, sei mehrfach auf sic eirgegangen. In ihren analytischen Parrien schließc die Studic an Habermas' Einwände' ${ }^{19}$ gegen die These vom Anbruch der Postmoderne an ${ }^{20}$ und incerpreciert den Strukcurwandel der gegenwärtigen Gesellschaft als einen Prozeß, in dem die Moderne die Halbheiten der industriegesellschafdichen Modernisierung hinter sich $\mathrm{lä}_{\mathrm{a}} \mathrm{t}$, indem sie eine weitere rcflexive Modernisicrung auf die bisherigen Prämissen der industricgcsellschaftichen Modernisierung anwendet. Im Gegensasz zu vielen irrationalistischen Fluchtwegen aus der Komplexitär der gegenwärtigen Sicuation uncerstellt und favorisiert sie cinen historischen Entwicklungsschub, der nicht die Beendigung der Aufklärung, sondern deren Einlösung gegen die bisherige Industriegesellschaft erfordert." Aus dieser Perspektive wird jener Werckonscrvatismus mancher Umweltschurzbewegungen, der sich umstandlos aus dem Bedürfnis zur Konservierung der Natur ableiter und ohne Unterscheidungsvermögen die normativ-demokrarischen Konzepte der Aufklärung zusammen mit der Aggressivitäi der wissenschaftlich-technologischen Entwicklung verwirft, seines schlichren lritums überführt. Andererseirs bleibr zu iragen, ob Becks Analyse der gegenwärigen Situarion nicht einen zu deudiclien Trend uncerstellt und ob ihre Hoffnung, daß wdie andere Gesellschaft viellcicht ohne Plan, Abstimmung und Bewußtsein aus den Werkstätren der rechnisch-ökonomischen Entwicklung entsteht ${ }^{22}$, begründer ist.

Die Frage, ob die Annahme eindcuriger Gerichtetheit der gegenwärrigen Entwicklung im Sinne fortaufender Modernisierung berechtigt ist oder durch die Beobachrung relevanter Rcfeudalisierungen zumindest korrigiert werden muß, cntstcht bereirs bei der Einschärzung von Einzelelementen der Gesellschaft, wie der veränderten Bedcutung von Klassenstrukiuren. Becks These lautet, daß im Zuge reflexiver Modernisierung die industriegesellschafrliche Symbiose von Stand und Klasse aufgelösc werde, indem eincrseizs ständische Subkulturen weggeschmolzen und zugleich andererseits grundlcgende Merkmale des Klassencharakters generalisiert werden. ${ }^{23}$ Nun ist unbestreitbar, daß die Gruppe der Lohnabhängigen immer größer

18 Uirich Beck, Risikogesellschatt. Aul dem Weg in eune andere Moderne. Frankfurt/M. 1986.

19 Jurger Habermas. Die Neue Unübersichılichkell. Kleıne poliusche Schrifun V. Frankluru/M. 1985, S. If) If. et parsim. Ders., Der philosophische Diskurs der Moderne, Frankfurs/M. $19^{85}$.

20 Ulrich Beck, Risikogesellschaft (Anm. 18), S. 12 ff., 31 i.

21 So auch Ulnch Beck, Gegengifec. Dic organisierte Unvernntwortichkert, Franklur/M. 1988, S. 291.

22 Uirıch Buck, Risikogesellschaft (Anm. 18), S. 307.

23 Ebd. 5 1 54 
wird, und daß an dic Tatsache der Lohnabhängigkeit als solcher ständische Solidariräı und lebensweltliche Evidenzen sich nicht mehr anlagern können. Andcrerseits verkennt der Hinweis auf die nivellierende Wirkung des wRisikos erwa durch eine so weit gestreute Arbeitslosigkeic, daß diese prinzipiell als Phase innerbalb jeder Einzelbiographie auftreten kann, die zu neuen Parzellierungen in dieser Generalisierung führt. Sie ergeben sich nicht nur aus der Gleichzeitigkeit von breiter Streuung und sozialer Strukturicrung der Arbeitslosigkeit ${ }^{24}$, sondern auch aus deren höchst ungleichen Auswirkungen. Zwischen den Betroffenen existiert eine scharfe Schranke je nachdern, ob finanzielle Reserven und Ressourcen mobilisiert werden können oder lebenszersiörende Konscquenzen trotz biologischen Überlebens zu befürchten sind. Zwar läßt die oft beschriebene Individualisierung durch Arbeicslosigkeit keine Bindung unter den so oder so Betroffenen aufkommen, aber die wirklich Betroffenen erleben das neue ständische Prinzip in negativer Form: den Ausschluß von allen Nichebetroffenen als Abbruch ibrer eigenen Kommunikationsfälxigkeit. - Die ncuc Parzetlierung durch generell drohende Arbeitslosigkeit zeigr sich aber auch in den Chancen, ihr zu entgehen. Beck selbst spricht angesichts der Tassache, daß gegenwärrig das Überangebor von formal Gleichqualifizierten zum Rückgriff auf Auswahlkriterien führt, in denen die Zuweisung nach Alter, Geschlechr, Auftreten", "Bezichungen«, kurz: "Herkunft" dominiert, von einer Renaissance wsändischer Kriterien und einer "Refeudalisierung * in der Verteilung gesellschaf(ticher Chancen. ${ }^{25}$

Erst rechr führe die steigende Professionalisierung und Spezialisierung zu neuen ständischen Verfestigungen. Auch sie müssen nicht unbedingt zu einer neucn gruppeninternen Solidarität führen. Auch hier sind die Grenzen eher negalı markiert und bezeichnen zumindest Behinderung von Kommunikation. So findet sich bercits in Musils Roman "Der Mann ohoe Eigenschafen «, der zugleich als große Gesellschaftsanalyse gelesen werden kann, die Darstellung einer Abendgesellschaft, auf der Spczialiscen verschiedener Fachgebiere als Gesprächsgegenstand nur noch das Wetter bleibr. Max Webcr betont in seincr Analyse der steigenden Berufsdifferenzierung und deren Konscquenzen in zunehmenden Partikularrechten und Partikulargerichtsbarkeiten durchaus die spezifische Modernität dieser Erscheinungen. ${ }^{26}$ Er behandelı sie nichr als Relikte alter ständischer Traditionen"7, sondern als Formationen einer ncuen Refeudalisierung, ohne ihre modernen Momente anders zu besrimmen als durch den Hinweis auf die Kombination von zweckrationaler Zugehörigkeit und formaler Abgrenzung, die jedenfalls Allgcmeinheit und Formalismus des modernen Rechts im Ganzen in Frage stellt. Es scheint aber, daß darüber hinaus ein wesentliches Charaktcristikum der Binnenstruktur und Außenbeziehungen der neuen ständischen Gruppierungen in dem typisch modernen Sachverhalt besteht, daß Individualisierung und Parzellierung zusammenfallen. Es ist der gleichzeitigen Atomisierung der Individuen in den Gruppen zu verdanken, daß die negatorsche Ausgrenzung zwischen den Gruppen zum dominanten Kriterium der neuen Refeudalisierung wird. Dics ist ein Phänomen, das für die Einscläazung neuer Mögliclskeiten demokratischer Organisation und für die Rekonstruktion des Prinzips der Volkssouveränitär in einer grundsätzlich parzellierten Gesellschaft von großer Bedeutung sein wird.

Auch daß die gegenwärtige Phase der Moderne übcrhaupt und auch hinsichelich

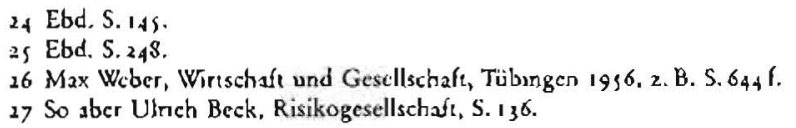

17 So aber Uirich Beck, Risikogesellschadt, S. 136 . 
ihrer politischen Integrationsmechanismen als eine der Refeudalisierung beschrieben werden kann, wirft für dic vorliegende Fragestellung Probleme auf. In den politisch-gescllschaftlichen Entscheidungsprozessen der Gegenwart haben nicht nur neokorporatistische Verflechtungen von Staats- und Wirtschaftsbürokratien das politische Entscheidungsmonopol unterlaufen, sondern in den wichtigsten Regelungsbereichen v.a. der Umweltpolitik sind die Handlungen policischer, wissenschaftlicher und ökonomischer Akteure in einer Weise vernetzt, die den Begriff der Entscheidung überhaupe in Fragc stellr. Damic scheinen konservative wie linke Souveränitärskonzepte am Ende zu sein. Jeder Dezisionismus nimmt angesichts unüberselibarer Abhängigkeicen von gesellschaftlichen Kreisläufen, unbeabsichtigter Nebenwirkungen und deshalb norwendiger Nachsteuerungen den Charakter eines ins Systemische überserzeen newigen Gesprächse ${ }^{28}$ an. Andererseits verlierr, rvas als Entscheidung nicht melir verantworcet werden kann, die Beziehung zu jeder Art von Volkssouveränitäc. In jedem Fall ist durch die gegenwärtige Vernetzung aller Handlungsbereiche jene historische Entwicklung wieder rückgängig gemacht, die bekanntlich Marx als Differenzierung von Staat und Gesellschafi beschricben und mic der Aufhebung der "Feudalicär " identifiziert hatce. ${ }^{29}$ Historisch scheinen Souveränitär wie Volkssouveränität an dicse Doppclung von Staat und Gesellschaft gebunden zu sein. Während der Absolutismus Souveränität und Gewalrmonopol auf seiten des Staates gegen die Gesellschaft konzentrierte, lokalisierte das liberale Demokratiemodell das politische Gewaitmonopol an der Spitze des Staates und Volkssouveränität an der Basis der Gcsellschaft in der erklärten Absicht, jeden Einsatz politischer Macht vom Willen an der Basis abhängig zu machen. Als Laski 1917 angesiches der Pluralisicrung der modernen Gesellschaft das Ende der Souveränitäı diagnostizierre ${ }^{30}$, hatte er allerdings eine Staatssouveränität bzw. Staatsorgansouveränität im Blick. Der Staat im ganzen bzw. das Parlament könne nicht mehr souverän sein, wenn der politische Sektor nur noch eine der gesellschafulichen Gruppen sei, mit denen er auí gleicher Ebene um den Einfluß auf politische Entscheidungen konkurriere. Die ratsächlichen Herrscher werden Laski zufolge wunsichtbar", wenn politische Entscheidungen und Parlamentsgeserze Ergebnis eines riesigen Komplexes aus Kräften innerhalb und außerhalb des Staates sind.

Wenn auch Laski das Problem noch nicht auf seinem jerzigen Enrwicklungsstand beschreiben konnte, so unterläuft ihm doch eine Vcrwechslung, die deshalb interessant ist, weil sie auch für gegenwärtige Verabschiedungen der Souveräniträtskategorie typisch ist. Laski identifiziert Souveränität, die er ja im Gegensatz zum ursprünglichen Demokratiekonzept im politischen System lokalisiert, weitgehend mit jener Einheit des Staates, die allen staatsmetaphysischen Annahmen zufolge als Entscheidungszentrum wic als unfassendes Ganzes zugleich die Einheit der Gesellschaft garanticrt und deren Teilmomente in sich integriert. "Erst diese Identifizierungen führen dazu, daß Laski die in jeder repräsentativen Demokratie banale Selbstverständlichkeit, daß Entscheidungen des Parlaments Ergebnis der Einwirkungen gesellschaftlicher Kräfce sind, bereits mic dem Verschwinden von Souveränität verwechselt. ${ }^{2}$ Wenn auch das Parlament nicht geradezu als Instrument der gesellschafulichen Basis gelten kann, so hatte doch schon Locke immerhin formuliert, daß nur die Zustimmung der Gesellscliaft der Parlamentsentscheidung den

2S So bezeichneı Carl Schmut den Gugenpol zur Entscheidungstonzcption, s. Poliuschs Romaneik, 2. Aufl. Munchen/Leipzig 192$\}$.

$29 \mathrm{Karl} \mathrm{Marx,} \mathrm{Zur} \mathrm{Judenfrage,} \mathrm{MEW} 1,5.367 \mathrm{t}$.

30 Harold J. Lasku, Studies in the Problem of Sovareignty, New Haven, London, Oxford igiz, S. rof.

31 Ebd. S. iff.

12 Ebd. S. 10. 
Charakrer eines Gesetzes verleihe." Es scheint, daß auch die lortgeschrittensten Diagnosen der Gegenwart Laskis Konnotation von staatlicher Einheit und Souveränität nicht ganz entgangen sind.

Gegenwärrig stellt sich längst nicht melır nur das Problem, daß der „Staat « nicht die Einheit der Gesellschaft garanticren karn, sondern daß er seine eigcne Einhcir verliert. Er konkurriert nicht wie eine identifizierbare gesellschaftliche Gruppe mit anderen Gruppen, sondern läßs sich von diesen kaum noch analycisch trennen. Laskis Nominalismus in der Beschreibung des Staates müBte angesichts dieser Situation überboten werden: Was wir noch als Staat bezeichnen, ist das jeweilige Ergebnis der Kommunikation zwischen verschiedenen fragmentierten Staatsapparaten, die ihrerseits informationsabhängig mit ihren gescllschafelichen "Umwelten a untrennbar verneczt sind. Ulrich Beck hat die Konsequenzen dieser Entwicklung für die "Risikoproduktion* der gegen wärrigen Gesellschaft glänzend analysiert: *Der Systeminterdependenz der hochspezialisierten Modernisierungsakteure in Wirtschaft, Landwirsichaft, Recht und Politik entspricht dic Abwesenheit von isolierbaren Einzelursachen und Verantwortlichkeiten *. Systemische Vernerzungen und "Schädigungstercisłáufe» greifen bei der Zerstörung der Natur so ineinander, daß jeder Akteur zugleich als *Ursache und Wirkung und damit Nichtursache" erscheint. Handlungssysteme nehmen so ihrerseits den fatalistischen Charakter des Ablaufs eines natürlichen Verhängnisses an. ${ }^{34}$ Entsprechendes gilt Beck zufolge fiir die Steuerung gesellschafticher Entwicklungen insgesamt. In Gewand von "unpolirischen * Investicionsentscheidungen wird über die ökonomische Verwertung wisscnschaftlicher Ergebnisse in einer Weise mirentschieden, daß sie die Allgemeinheit in höchst politischer Weise betreffen und niche nur künftige gesellschaftliche Entrvicklungsperspektiven vorzeichnen, sondern möghicherweise über Art und Weise bzw. Fortbestand des Lebens überhaupt bestimmen. Diese Entscheidungen der wissenschaftlichen, technischen und ökonomischen "Subpolirik * befinden sich * jenseirs demokratischer Zustimmung*, sie haben in diesem Sinn «keinen Orew, an dem sie sichrbar gemacht und diskursiv erarbeitet werden könnten. Auf diese Wcise schließen sich die nUnerkennbarkeit a und "Unverantwortbarkeita der Folgen und die "Nichrentscheidbarkeita des technischen Fortschritrs" zur "Niemandsherrschaft w der Risikogeselischaft zusammen."

Mit diescr Analyse der faknischen Abwesenheit von Souveränicät in der gegenwärtigen Gesellschaft, in der überlebenswichtige Entscheidungen, die die systemischen Schadenskreisläufe unterbrechen könnten, gerade nicht getroffen wcrden, ist offensichrlich der Kern der gegenwärrigen Problemacik auf den Begriff gebracht.

Umso prekärer erscheinen darum Hoffnungen, daß das gegenwärige Stadium der Gesellschaft Pcrspektiven zur selbstüligen Entwicklung einer "anderen " rationaleren Gesellschaft in sich enthalte - Hoffnungen, die gerade an den fakcischen Souveränitärsverlusı anschlicßen. Die Überzeugung, daß das neofeudale Verschwinden der "Politik" in dezentralen "Subpolitiken Demokratisierung enthalte ${ }^{16}$, setzt die gleiche falsche Identifikation von Souveränität und mystischer Einheit eines staatlichen Entscheidungssubjckes voraus, wie sic ungekehrt auch antidemokratischen Lösungsvorschlägen im Krisenszenario der

3) John Lacke, Two Trealises (Anm. S), \$ 1 s4

34 Ulrich Beck, Risikogesellsschafe (Anm. 18), bes. S. 4 i i

35 Fbu. S. 253,30 f.

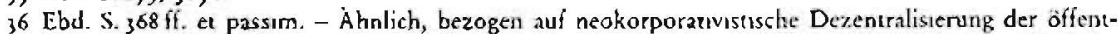
lichen Verwaleung: Karl-Hein' Ladeur, wAbwägunga - enn ncucs Rechesparadigma? Von der Einheie der Rechesordnung zur Pluralita der Rechtsdiskterse, in: Archiv fur Rechts- und Sozıalphilosophie 69 $(1983), 5.463$ if 
Gegenwart zugrundeliegt. Von sozialistischen Entwürfen cincr Ökodiktatur bıs zu rechtskonservativen Konzepten, die angesichts des ökologischen Ausnahmezuseands" darauf dringen, daß die autonome polizische Enrscheidung über den technologischen Prozeß nur zurückzugewinnen sei, wenn man auf den Luxus von Demokracie und Rechrsstaat verzichce ${ }^{37}$, reicht die populäre Konnotation von Souveränität und staatlichem Entschcidungsmonopol.

Die basisdemokratische Variante der Diskussion über Entwicklungsperspektiven der gegenwärtigen Gesellschaft neigt deshalb zu dem Fehler, die Qualität jener Dezeneralisierung zu vernachlässigen, an die sie ihre Hoffnung auf Demokrarisierung knüpft. Nicht jede Dezentralisierung hat aber den gewünschten Effekt. Angesichr der pluraliscischen Konkurrenz dezentraler Machckomplexe und der weitgehenden Delegation öffentlicher Aufgaben an Private hauc Franz Neumann sogar das NS-System als einen Nicht-Staat beschrieben, weil es (entgegen der "Fülirerstazts $\alpha$-Ideologic) eines einheitlichen Entscheidungszentrums entbehre. ${ }^{88}$ Wic dicses extreme Beispiel zeigr, kann die *Entmachtung von Politiku zugunsten ihrerseits vermachteter Teilsystcme zu einer Situation führon, die es anderen machtlosen Subpolitiken noch weniger gestatter, auf die Entscheidungen der dezentralisierten Machrkomplexe Einfluß zu nehmen, als es auf dem Umweg über eine funkrionierende policische Zentrale möglich ist. Aus der Perspektive des klassischen liberaldemokratischen Konzepts kann insofern die Encmachtung der Policik auch als Zerfall cines Zentrums wahrgenommen werden, das die Aufgabe hätre, Lernprozesse, die an der gesellschaftichen Basis z. B. in bezug auf Unweltzersiörung gemacht worden sind, in Entscheidungen gegen die gesclischaftlichen Machtsysteme der tcchnologischen Entwicklung umzusctzen. Eine Restauration dieses Zentrums im klassischen Sinne ist aber weder möglich noch aus basisdemokratischer Perspektive wünschenswert. Insofem geht der Streit zwischen den herrschenden Alternativkonzepten und der hier vorgeschlagenen Rekonstruktion der Volkssouveränität um dic denokratische Qualität der Dezentralisierung und um die Frage, auf welche Weise die machtlosen Subpolitiken auf diejenigen Subpolitiken Einfluß nehmen sollen, die heute die Entwicklungsperspektiven der Gesellschafr bestimmen. An dieser Konraktstellc, an diesem gesellschafulichen "Orta liegt jedenfalls heute das Problem der abwesenden Volkssouveränität. Nicht von deren Realizät kann also hier die Rede sein, sondern nur von deren möglicher Realität.

II.

Ein Versuch zur Rckonstrukcion von Volkssouvcränität unter diesen Bedingungen muß von der fakrischen gesellschafelichen Parzellierung ausgehen, kann sich aber nicht mit der schieren Beschreibung dessen zufriedengeben, was als politischinscitutionelle Umsctzung dieser Entwicklung gegenwärtig der Fall ist. Die umstandslose Anpassung des insticurionellen Rahmens an die gesellschaftlichen Veränderungen bestehe darin, ausschließlich dezentralisierte Konflikıbearbeitungsmechanismen zu fordern. So richten sich die Hoff́nungen vieler alternativer Konzepte seltsamernveise auf die Gerichte, als könnten diese zu den gesuchten Umschaltstellen

37 Die letzere Option ist z. B. für enschlagige Publikationen im Seewald Verlag besonders typisch.

38 Frane Nevmann, Behemoth. The Structure and Praccice of Narional Socialism 1935-1944. 2. Aufl. New York : $y_{3}$. Vgl. zu diesem Problem Ingeborg Maus, Bürgerliche Rechtstheorie und Faschisnius. Zur sozalien liunkuon und akiucllen W'irkung der Theone Carl Schmus, 2. Aufl. Munchen ig80, S. I 66 ff, ig2 if. 
2wischen basisdemokratischen Lernprozessen und vermachrecen Subpolitiken avancieren. ${ }^{39}$ Aus der Fetischisicrung politischer Dezentralisierung als solcher ergibt sich auch hicr die Vorstellung, als sei die fakcische Entmachtung der parlamentarischen Zentrale zugunsten gerichtsförmiger Entscheidungen an der Peripherie selbst schon der Prozeß, der sich als Demokratisierung der Politik versichen ließe. So wird das neue Zusammenspiel zwischen Bürgeriniciativen und Gerichten in Kategorien beschrieben, die die klagenden Bürger zu politischen Partizipanten deshalb aufwerten, weil diese dic Justiz als Instrument gesellschaftlicher Grundbedürfnisse gegen den mir der Technostruktur verfilzten Staat einserzen ${ }^{40}$ - als bätte die Justiz über Nacht aufgehört, selber eine staatliche Agentur zu sein und, jenseirs einzelner unorthodoxer Entscheidungen, eher der Machtsteigerung als der Machtbeschränkung dominicrender Subpolitiken zu dienen.

Vor allem aber macht gerade die beschriebene Unübersehbarkeit komplexer Folgewirkungen innerhalb der neuen Risikogesellschaft solche Holfnungen unrealistisch. Auch wo Gerichte versuchen, z. B. durch die extensive Auslegung haftungsrechtlicher Bestimmungen, den an sie gerichreten walternativen* Ertvarzungen gereche zu werden", begeben sie sich in die unlösbar gervordene Schwierigkeit hinein, die Schädigung eines bestimmten Stückes Umwelt durch einen bestimmten Schadstoff im vorliegenden Einzel\{all zu prüfcn - während die überhaupt schädliche Wirkung des in Frage stehenden Sroffes hinlänglich bekannt ist. Es liegt gleichzeitig an der Struktur justizförmigen Enrscheidens, daß Gerichte, auch wenn sie guten Willens sind, hier nicht Abhilfe schaffen können. Da sie nur im bercits eingetretenen - oder bei Verwaltungsgerichtsentscheidungen: im gerade administrativ anvisierten - Einzelfall angerufon werden können, sind sie immer nur mit den Folgen längst getroffener ökonomischer Entscheidungen oder mir den (höchst unsicher) crwartbaren Folgen einer konkret ansrehenden Verwaltungsentscheidung befaßt. Ihre Art der Konfliktbearbeitung ist deshalb nicht nur dezentral, sondern, was hier das Wesentliche ist: reakttu. In dieser Festschreibung der Beweislast auf den Einzelfall, die also in der Struktur juriscischen Entscheidens selbst liegt, ist die grundsätzliche Machtlosigkcit der Gerichte begründet, wenn es darum geht, eine Richtungsänderung der gegenwärcigen Entwicklung einzuleiten. Woran es gegenwärtig fehlt, sind (nichı nocwendig zentralistisch gecroffene, aber) generelle Entscheidungen, wie z. B. schlichre Verbote überhaupt schädlichcr Stoffe, die den konkreten Konfliktfällen vorhergehen und auf die die Gerichre sich bei ihren Einzelentscheidungen erst beziehen könnten. Angesichts dieser Lage besteht die norganisierte Verantwortungslosigkeit " gerade darin, Umweltkonflikes auf die Justiz zu verlagern und ecwa auch durch parlamentarische Aufforderungen zur gerichelichen Klärung der rechtlichen Perspektiven eines Umweltproblems die Aufmerksamkeit der protesticrenden Bevölkerung auf diesen Nebenschauplatz abzulenken. Der Prozeß der Refeudalisierung, der bercits in der Abwanderung der Politik in mächtige Subpolitiken liegt, wird durch die Favorisierung der Justiz als dezentraler Schlichtungsinstanz noch verschärft. Auf diese Weise kommt es auch zu einer Refeudalisierung des gesamten Rechtssystems: Während der individuelle Mord, die handgreifliche Körperverletzung und die einzelne Tierquälerei wie eh und je den klassischen Verboren des

39 Zu dicsim Trend ausfuhrlicher Ingeburg Maus, Jusus als gesellschaltiches Uber-Ich. Zur Funkwon von Rechisprechung in der wineelosen Gesellschafı a, in: Werner Faulsuch, Gunter E. Grimni (Hg-), Siurz. der Göuer? Vaterbilder in Literatur, Medien und Kultur des 20. Jahrhundens, FrankfurvM. 1989, S. $121 \mathrm{il}$.

40 5. x. B. Karl-Heinz Ladeur, Vom Gesetzesvollzug zur strategischen Rechtsforibildung, in: Leviathan 7 (1979), S. 339ff., 349. Áhnlich auch Ulrach Beck, Risikogesellschaft (Anm. 18), S. 316.

4) Dayu 7. 13. Ger Brüggemeıer, Umwelthafrungsrecht - Ein Bettrag zum Recht der ×Risikogescllschafte?, in: Knessithe Jusiz $22(1989), 213$ fl, 
Rechts unterliegen, errichre dic lediglich gerichtsförmige Einzelfallbearbeitung von Umwelıschädigungen für dic millionenfachen Täter wahrhaft feudale Privilegien ${ }^{42}$, die den Universalismus des modernen Rechts ganz grundsäızlich außer Kraft setzen.

Die Überlegung, wic angesichts dieser gegenwärtigen gesellschaftlichen Problemlage Chancen der Einwirkung basisdemokraxischer Lernpotentiale auf subpolitische Machtkomplexe zu cröffnen seien, kann sich darum nicht mit dem Hinweis auf die fakrisch sceigende Inanspruchnahme der Rechesprechung beruhigen, sondern muß sich auf die Recheretzung konzentrieren. Eine Rückwendung der Aufmerksamkeit auf die Gesetzgebungshunktion ist nicht - wie das überaus populäre Vorurteil cs will - identisch mic dem (hoffnungslosen) Versuch der Wicderherstellung eines einheitlichen politischen Zentrums. Eine Rekonstruktion von Volkssouveränität in einer parzellierten Gesellschaft muß vielmehr cine Dezentralisierung der Gesetzgebung ins Auge fassen, wälırend sic die generalisicrenden Perspektiven der Rechisform beibehält. Sie ist Rekonstruktion insofern, als auch die klassische Demokrarietheorie der Aufklärung Souveränität als Geserzgebungskompetenz definierce und Volkssouveränität mit dem wie immer institutioncll gesicherten Recht des Volkes gleichsetzte, nur solche Gesetze anzuerkennen, die es selbst gesetzt hatte. ${ }^{43}$ Der Vorschlag einer so bestimmten Anverwandlung des klassischen Konzepts wird also dessen drei zentralen Komponenten höclist unterschiedlich behandeln: Während der Zentralismus der Rechrserzung nicht mehr aufrecherhaiten werden kann, bedarf Volkssouveränität als »Seibstgcseizgebung des Volkes« überhaupt erst der Verwirklichung. Das cinzige Bleibende im Wechscl wäre die (freilich auch modifizierte) Generalität der Rechisform.

Letzterer hafter - um damit zu beginnen - hinsichtlich der Umweltpolicik tatsächlich ein nostalgisches Moment an, als sie unverändert ein zentrales Organisationsprinzip der Ökonomie in Rechnung stellt. Das liberale Demokraciemodell hatce sich bekanntlich mit der Forderung der Gcneralität des Gesetzes verbunden, um unter andercm staatiche Eingriffe in die Wirtschaft für diese berechenbar zu halten und die Gleichheit der Wetrberverbsbedingungen zu sichern. Auf diese Weise waren auch politische Eingriffe nach sozialen, also wirtschaftsfremden Gesichtspunkten möglich. So war dic sZehnstunden-Bill« nur als generelle. Regełung ökonomisch verträglich, aber auch überhaupt durchsetzbar: Jeder einzelne Unternehmer wäre im Konkurrenzkampf unterlegen, der sie individucll eingcführt häcte. Was das Verbol cines Umweltgif́s von der "Zcinnstunden-Bill « unterscheidet, ist die Tatsache, daß

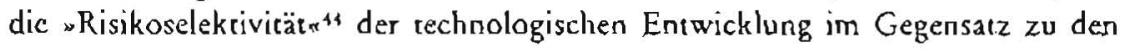
betrieblichen Pauschalbelastungen von Sozialgeserzgebung zu ganz unterschiedlichen Konsequenzen für einzelne Branchen führt. Dennoch betrifft das Verbor eincs Schadstoffs alle potentiellen Hersteller noch immer in gleicher Weise. Hatte die frühe Demokratietheorie noch eine extreme Gcneralität des Gesetzes begründet, die wirklich "alle gleichermaßen betreffen sollte, und damit politische lnerventionen in die Gesellschaft aul das Minimum des gemeinsamen Interesses beschränkr", so stößc die Generalität des Gesetzes gegenwärtig auf immer weniger Betroffene -

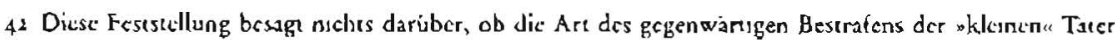
vertretbar ist, sondern beschränkr sich damuf, die extreme Ungleschheic in der heurigen Rechespraxis zu bezachnen.

4) John Locke, Tivo Treauses (Anm. 5), \$1 49, 134 fi.; Rousseau, Du Contrat social (Anm. S), II 1, 2 und 6; Kant, Metaphysik der Sitten. Rechtslchrc, $\$$ 4s und 46. Werkausgabe Bd. 8. hg. von Wilhelm Weischedcl, FrankfurdM. 1977, S.431f.

44 Ulrich Bcck, Risikogesellschaft (Anm. 18), S. 367.

as Dies ist - entgegen vicler Irriumer über esne ingeblich vtouliearcu Demokrauekonzeption - auch die Incersion Rousscaus, s. Contrat social II, I Abs. 1. 
und dies nicht nur im Umweltbereich. Auf jeden Fall ist die generelle Rechtsform mit gesellschaftlicher Parzellierung komparibel.

Andererseits muß das generelle Verbot nicht notwendig zentraliscisch erarbeiter worden sein. Gerade die politische Zentrale der Rechtsetzung ist gegenwärtig durch den privilegierten Zugang der stärksten Umweltschädiger okkupierı, deren Einfluß auf die Geserzgebung sich weniger in der Durchsetzung bestimmter, sondern möglichst unbescimmer Regelungen bemerkbar macht. Das allseirs beklagte Implementationsdeficit ist darum nicht einfach der Komplexitat gegenwärriger Umweltbedingungen anzulasten, sondern durch diese herrschende Tendenz zur Entregelung trotz steigender Verrechrlichung beding ${ }^{4}{ }^{\natural}$, die noch immer in dem Drohporential von Investitionsentscheidungen, Standortwahl, kurz: ökonomischer Machr begründet ist. Die Informationsabhängigkeir der politischen Zentrale von mächtigen Subpolitiken kann darum nur durch dezencrale Informicrcheit vor Ort unterbrochen werden. Insoferm sind die in der Tat hoffnungsvollen Ansätze z. B. innerbetrieblicher Opposition beim Einsarz neuer Technologien und ihre informarionsvermittelnde Kooperation mic basisdemokratischen Tcilöffentlichkeiten und Protestbewegungen in einer Weise zu koordinieren und mit rechelich gesicherten Einflußchancen auszustatten ${ }^{47}$, daß sie nicht wirkungslos in der Einleitung von "Rechtswegen * verpuffen, sondern Rechrsänderungen bewirken können. Eine Rekonstruktion von Volkssouveränicät, die die Möglichkcit einer "Selbstgeserzgebung des Volkes« untersucht, wird sich dabei nicht mit eher zufälligen Ergebnissen äußeren gesellschaftlichen Drucks auf die "policische Zentrales zufriedengeben können. Sie vertraut niche darauf, daß neue Formen der Koordination aus der gegen wärtigen gesellschaftlichen Situation "ohne Plan, Abstimmung und Bewußssein* hervorgehın, sondern sucht nach neuen Möglichkeiten einer gesellschaftsadäquaren Institutionalisierung der Rechtsetzung, dic die vorhandenc parlamentarische ergänzt.

Weiche gesellschafelichen Regelungsbereiche - das Umweltproblem steht hicr nur als cin besonders wichtiger pars pro toto - durch dic gesellschafelich beeinflußtc parlamentarische Zencrale entschieden und welche besser dezentralcn Rechrsetzungsprozessen überlassen werden sollten, kann nicht im Detail vorgeschlagen werden und unterliegr koliekriven demokratischen Reflexions- und Enrscheidungsprozessen. Angesichts der Tatsache, daß die Generalität des Rechts gegenwärtig vielfältigen Brechungen einer parzcllierten Gesellschaft unterliegt, bieter sich aber einc Arbeitsteilung zwischen Zentrale und Peripherie nach dem Grad der Anwendungsallgemeinhcit ciner Rechisregulierung an. ${ }^{48}$ Rechtsnormen, die sich nur an eine sehr begrenzre Zahl von Adressaten richren oder nur regionalc Ausivirkungen haben, können in Rechtsetzungsarrangements beraten und verabschieder werden, in denen die betroffenen Konfliktparteien einander direkr konfroniert und mit sy'mmetrischen Verhandlungspositionen ausgestattet werden, die die Asymmetrien gesellschaitlicher Macht rechtlich kompensieren. Rechrsnormen, die nahezu oder tarsächlich "alle "betreffen, könnten dezcntral vorbereiter und zentral koordinicrt und verabschicdet werden. Alles dies setzt voraus, daß die parlamentarische Zentrale für die allgcmeinste Funkrion zuständig bleibr: die Setzung von Verfahrensnor-

${ }_{4} 6$ Peter Knoeplel, Verreclitlichung und Interesse. Interessenberücksicheigungsmusecr in dres Grundiypen von Verrechulichungsstrategien aus der Unwelt-, Risiko- und Bildungspolitik, in: Rüdiger Voigt (Hg.), Verrechlichung, Königstein/T's, 1980 , S. 77 fl., 84; Ders./H. Weidner. Normbildung und Implemencatıon: Interessenberüctesichtigungsmuster in Programmstrukturen von Lufteeinhaliepolitiken, in: Renate Maynix (Hg.). Implementation poliuscher Programme, Könıgsten/Ts. 1980. S.82 ff., 101.

47 So auch Ulrich Beck, Risikogesellschaft (Anm, 18), S. 372 f., wenngleich mit anderen (justizorienescren) Ronsequenzen.

$4^{8}$ Dazu cinge Andcutungen be Ingeborg Maus, Rechisihcosic und politische Theorie in Industriekapitalismus, Munchen 1986, S. 297 ff. 
men, nach denen in dezentralen Rechtsctzungsprozessen die inhaltlichen Normen erst zustandekommen. Dies nicht, weil noch eine Einheidlichkeit der proxeduralen Normen (zentral) garantiert werden müßte, sondern weil nur eine institutionelle und zeitliche Differenzierung zwischen "Normicrungen der Normserzung und den Normsetzungen sclbst ${ }^{* 9}$ auf einer neuen Ebene jenc Invarianz von Verfahrensnormen in bezug auf den konkreten Fall garantieren kann, die aus rechtsstaatlichen Gründen unerläßlich ist: Ebenso wie nach dem klassischen Konzept (belastende) inhaltliche Rechesnormen belicbige Diskriminierungen und willkürliche Durchgriffe auf einzelnc Personen und Gruppen nur dadurch verhindern können, daß sie für unbestimmt viele zukünftige Fälle formuliers sind, so ist auch von Verfahrensnormen nur dann ein Mindestmaß an Fairneß zu crwarten, wenn bei ihrem Zustandekommen der jerveils konkrete gesellschaftliche Interessenkonflike noch nicht bekannt ist, der nach Maßgabe ihrer Positions'zurweisungen ausgetragen werden soll. Diese Reflexivität in der Trennung zwischen prozeduralen Entscheidungsprämissen und inhaltlichen Entscheidungen könnce in der neuen Arbeitsteilung zwischen zentraler Verfahrensgesetzgebung und dezentraler inhalticher Gesctzgebung jene Unkenntnis des konkreten Falles wiederherstellen, die in der gewaltenteiligen Abstufung rechtsstaatlicher Entschcidungsverfahren einmal bestand und heute durch die faktischen Refeudalisierungen des politischen Systems längst nicht mehr garanciert ist.

Dieser Vorschlag zur Demokratisierung der Geserzgebung nimmt gleichzeitig darauf Rücksicht, daß eine Allgemeinheit des Interesses angesichts der gesellschaftlichen Parzellierung nicht mehr im Inhaltlichen gefunden werden kann. Die Dominanz des Negativen in den gegenwärtigen Gruppenbeziehungen, also die Tatsache, daß negatorische Ausgrenzungen solidarische Binnenstrukturen an Bedeutung überereffen, führr dazu, daß weder allgemeine noch besondere inhalcliche Forderungen auf Dauer gestellt werden können. So ist es für heurige politische Organisationsversuche an der gesellschaftlichen Basis eher typisch, daß sie zu Gruppenbildungen anläßlich sehr spezifischer single issues führen, die Bündnisse mit anderen single issue-Bewegungen nur gelegentlich eingehen und selbst wieder zerfallen, sobald ihre Anliegen administrativ entweder positiv beschieden oder definiriv entmutigt worden sind. Politische Allgemeinheit kann deshalb nur noch hinsichtlich der Verfahren definicrt verden, innerhalb derer die Parrikularität und Siruativität der gesellschaftlichen Inhalte und materiellen Interessen zur Konsens- und Entscheidungsbildung freigegeben wird. Wre die Bescimmung eines universaliscischen Moralprinzips sich auf das immer differenziertere $n$ Besonderc der modernen Gesellschaft nur noch "indireke "beziehen kann und deshalb eine ausschlicßlich prozedurale Fassung annimmt ${ }^{\circ 0}$, so wird auch eine gesellschaftsadäquate Institutionalisierung demokratischer Rechisetzung sich auf den Aspekt des Verfahrens konzentricren müssen. In diesem Sinne zwingt die gegenwärtige Situation - allen Wertsubscantialisicrungen verbreiceter Verfassungsjudikatur zum Trocz - schon längst zu einem Verfassungsbegriff, der sich nicht an der Fixierung vorentschiedener Inhalte oricntiert, sondern die Verfassung als Festlegung von Verlahren begreift, in denen überhaupt erst über Inhaire entschieden wird." Diese reflexive Ausdifferenzierung zwischen Verfasiung und Gesetzgebung wird nur aul die nächste Stufe weiterverlagert, wenn es zu einer Arbeitsteilung innerhalb der Gcsetzgebung kommt. Den dezentralen und parzellierten inhaltlichen Enrscheidungen licgr das zentral entschiedene Prozedere voraus.

49 Ebd. in Abwandlung der Pramissen von Niklas Luhmann.

so Jürgen Habermas, Moralicat und Sivelichke'te (Anm. 16), S. 16 ff., bes. 26.

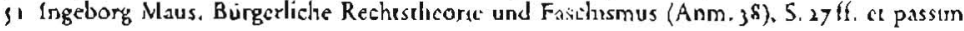


Dessen Ausgestaltung und Entwicklung kann deshalb die allgemeine Aufmerksamkeit und politische Beteiligung aller gesellschaftlichen Gruppen finden, weil es Prämisse iltrer eigenen besonderen Entscheidungen ist.

Dic klassischen Theorien der Volkssouveränität, dic die prozedurale Selektion inhaltlicher Rechrsentscheidungen ausschließlich von zentralen Geserzgebungsprozessen errvarteten, hatten das Problem der Enffremdung dieser Entscheidungen von der gesellschaftlichen Basis in die Formel gekleidet, daß hier das Volk als Einheit über das Volk als Vielheit herrsche. ${ }^{\text {s2 }}$ Daß es "das Volk« so wenig gibt wie den Staat, ist nicht neueren Entwickłungen zu verdanken; es war, wie diese Formulicrung ausweist, schon immer ein fiktiver Begriff. Aber erst eine Freisetzung autonomer gesellschaftlicher Gesetzgebungsprozesse, in der die partikularen Interessen zu ihrem je spezifischen Rechr kämen, könnte die Fiktivitär dieses Volksbegriffs in einer Weise steigern, die dessen progressive Momence sichrbar werden ließe. Indem der Zwang zu inhaldticher Einheit in dezentraler Autonomie verschwindet, bleiben nur noch die abstraktesten ciroyens ais Substrat dieses Volksbegriffs zurück. Worauf man sich noch einheizlich cinigen muß, hat mit den Unterschieden von Herkunft, Geschlecht, Rasse, Religion und Kultur nichts zu cun. In den Bestimmungen der Sraats- und Aktivbürgerschaft hatce sich die Französische Revolution diesem Volksbegriff lediglich angenähert. Aber erst in der völligen Abscraktion von allen inhaltlichen Momenten ist ein enttraditionalisierter, postkonventioneller Volksbegriff gewonnen, der sich mir einer pluralisierten und multikuiturellen Gesellschaft verträgr. Die klassische Idee der Unteilbarkeit der Volkssouveränitär verweist unter diesem Aspekt nicht auf ein mystisches Kollektivsubjekt, sondern enthält die schlichre Forderung, daß Souveränirät ausschließlich denen zukomme, die von Entscheidungen selbst betroffen sind - und nichr etwa den Amtswaltern und Funktionären. Die Idee der Einhcit und Unceilbarkcit der Volkssouveränitär bezeichnet nichıs anderes als den \#Sraatu in den Händen des "Volkes“. 